\title{
Ist durch die Abrasio eine Danerheilung des beginnenden glandulären Uteruscarcinoms zu erzielen?
}

Von

\author{
Dr. W. Vassmer, \\ Frauellarat in Hamover.
}

(Herva Tafel KVII.)

So ketzerisch diese Fragestellung auch lauten mag, zumal Angesichts der modernen Forderung, jedes auch beginnerde Carcinom des Uterus der erweiterten abdominalen Radicaloperation iu unterziehen, so steht doch theoretisch der Möglichkeit einer Dauerheilung einer beginnenden und auf die Mucosa beschränkten epithelialen malignen Neubildung des Uterus durch die Abrasio nichts im Wege.

Ein sicherer Beweis für eine derartige Dauerheilung kann meines Erachtens allerdings nicht in den Fällen erblickt werden, in denen die mikroskopische Untersuchung des exstirpirten Uterus das nach der mikroskopischen Untersuchung der vorausgegangenen Abrasio supponirte Carcinom nicht nachweisen konnte. Denn bei unserer Unkenntniss bezüglich der Aetiologie der bösartigen epithelialen Neubildungen bleibt immer noch der Einwand bestehen, dass, selbst wenn zur Zeit auch keine earcinomatösen Veränderungen in der nach der Abrasio zurückbleibenden bezw. schon wieder regenerirten Mucosa nachweisbar sein sollten, es doch vielleicht früher oder später wieder von den mit carcinomatöser Disposition ausgestatteten epithelialen Elementen zu einer epithelialen bösartigen Neubildung gekommen sein würde.

Ein absolut sicherer Beweis für die Möglichkeit einer Dauerheilung des beginnenden Uteruscarcinoms durch die Abrasio kann meines Erachtens nur in den Fällen erblickt werden, in denen nach gestellter mikroskopischer Diagnose die Totalexstirpation aus irgend 
einem Grunde unterbleiben musste, die Patientin trotzdem gesund und frei von irgend welchen für Carcinom sprechenden Symptomen blieb und Wiederholungen der Ausschabung stets normalen histologischen Befund ergaben.

Aus leicht erklärlichen Gründen sind derartige Fälle äusserst selten. Um so mehr Interesse dürfte daher folgender Fall beanspruchen, in welchem die eben aufgestellten Forderungen für die Berechtigung der Annahme einer derartigen Dauerheilung nach Abrasio mehr oder weniger zutreffen.

Am 16. 11. 1901 erhielt ich mit der kurzen klinischen Notiz „45 j. Phthisica, Periode erst nach 5 Wochen eingetreten, - blutet seit 14 Tagen" vom Collegen Dr. A., Fravenarzt in E., durch Ausschabung gewonnenes Material zur mikroskopischen Untersuchung zugeschickt. Der erste, dem Collegen und auch mir vorschwebende Gedanke war natürlich, dass es sich trotz des Alters der Patientin wahrscheinlich um Blutung nach bezw. bei frühzeitig unterbrochener Sehwangerschaft handeln würde.

Um so überraschter war ich daher, folgenden mikroskopischen Befund erheben zu müssen. Schon makroskopisch lässt sich an den gehärteten und geschnittenen Präparaten eine starke Verdickung der. Mucosa constatiren, in dem einzelne Gewebsstücke bei senkrechter Schnittführung durch die Mucosa eine Höhe von $4 \mathrm{~mm}$ ergaben und mikroskopisch lässt sich aus der 3 seitigen Bedeckung einzelner Schnitte mit Oberflächenepithel, sowie aus der unregelmässigen Gestalt der Drüsen innerhalb dieser Partien auf eine zum Theil polypöse Form der Mucosawucherung schliessen.

Betrachten wir jetzt die einzelnen Gewebselemente der Mucosa im mikroskopischen Bilde, so zeigt zunächst das Stroma ein sehr wechselndes Verhalten. Meist ist es sehr zellreich, aus Rund- und Spindelzellen zusammengesetzt, von denen die Rundzellen häufig abnorme Grösse und verhältnissmässig häufig Mitosen zeigen. An manchen Stellen ist das Stroma weitmaschig und zeigt in diesem weiten Maschenwerk nur runde, beträchtlich vergrösserte Zellen; an ein er kleinen Stelle ist es diffus durchsetzt von gelapptkernigen Leukocyten. Vielfach ist der Reichthum des Stroma an Rundzellen so gross, dass entfernt Anklänge an Sarkom- oder wenn kleinste Capillaren Mittelpunkt dieser Zellvermehrung sind, an Endotheliombezw. Peritheliombildung entstehen. Wo in Folge polypöser $\mathrm{Wo}$ cherung der Mucosa das Stroma erbeblich verbreitert ist, sind die 
Capillaren stark erweitert und zum Theil mit hyalinen, von gelapptkernigen Leukocyten durchsetzten Thromben erfüllt.

Ebenso wechselnd wie das Verhalten des Stroma ist das der Drüsen. Während sie auf weite Strecken sich durch ihre Anordnung und Verlauf noch als Einstülpungen des Oberflächenepithels ausweisen und abgesehen von Schlängelungen, Invaginationen und Ausstülpungen normales Verhalten zeigen, ist in jenen hypertrophischen Partien diese Regelmässigkeit verwischt und man trifft hier neben langen, schmalen, die Mucosa in den verschiedensten Richtungen durchziehenden Gängen, weite dilatirte, epitheliale Gebilde mit zahlreichen peripheren Ausstülpungen einerseits, wie fingerförmigen schmalen peripheren Erhebungen in das Iumen andererseits.

Neben der Unregelmässigkeit in Grösse, Form und Verlauf der einzelnen Drüsen selbst, fällt bier auch eine gewisse Unregelmässigkeit in der Form der die Drüsen auskleidenden einzelnen epithelialen Elemente auf. Während das Epithel in einzelnen Drüsen einschichtigen, viedrig-eubisehen, fast endothelartitgen Charakter zeigt, wird es in anderen dentlich unregelmässig mehr.. schichtig, wobei zugleich die einzelnen Zellen sehr verschiedenartige, runde, ovale bis polygonale Kernformen zeigen. In vielen Drüsen wird eine Mehrsehichtung dadurch rorgetäuscht, dass die stäbchenförmigen Kerne der neben einander liegenden Zellen siek mehrzeilig gelagert haben; in anderen handelt es sieh aber um wirkliche Mehrschichtungen, indem neben und zwisehen den hoch-cylindrischen Zellen mit scharf gefärbten stäbehenförmigen Kemen rundliche Zellen mit hellen bläschentörwigen Kernen auftreten, die in unregelmässiger Schichtung übereinander liegen and sich ron den erwähnten cylindrischen Zellen wie auch von den viel kleineren durch das Epithel wandernden Rundzellen scharf unterseheiden.

Sehr auffallend ist gerade in den unregelmässig gestalteten, zum Theil stark erweiterten Drüsenlumina der polypös gewucherten Mucosa der plötzliche Wechsel des Epithelcharakters innerhalb ein- und derselben Drüse, indem plötzlich an Stelle đer einschiohtigen, ein- und mehrzeilig angeordneten hohen oylinderzellen mit scharf gefärbten stäbchenförmigen Kemen jene grossen runden Formen wit leicht bläschenförmigen Kernen auftreten, dio abgesehen von der Mehrschichtung, besonders auch durch die suht viel mattere Färbung (Alauncarmin) sich scharf ron den erwähnten Zellformen unterscheiden. Mitosen begegnet man in diesem un- 
regelmässig mehrschichtigen Epithel relativ selten, wie auch, $a b$ gesehen davon, dass um manche dieser Drüsen sich das Stroma zu dichteren Zügen spindeliger Bindegewebszellen verdichtet hat, jede Reaction von Seiten des umgebenden Stromas fehlt.

Auch das Oberflächenepithel zeigt stellenweise Uebergänge in unregelmässig mehrschichtiges Epithel; nirgends aber sieht man dieses mehrschichtige Oberflächen- und Drüsenepithel als solches in das umgebende Stroma eindringen, sondern überall ist die Grenze zwischen Epithel und Bindegewebe scharf erhalten.

Neben diesen wohl nur als gutartige, interstitielle und glanduläre Hypertrophie- und -Plasie zu bezeichnenden Wucherungsvorgängen der Mucosa fanden sich nun in einem ca. $2 \mathrm{~mm}$ langen und $1 \mathrm{~mm}$ breiten Mucosafetzen Wucherungsvorgänge an den Drüsen; die den Gedanken an eine beginnende, bösartige epitheliale Wucherung wachrufen mussten und mich demgemäss auch diese Diagnose dem Collegen Dr. A. gegenüber stellen liessen.

Schon bei schwacher Vergrösserung hebt sich diese, ganz isolirt für sich liegende, Mucosaparthie durch ihre matte Färbung und durch die eigenartige Anordnung der epithelialen Elemente scharf von dem übrigen Mucosagewebe ab. Statt der normalen Drüsenlängsund -querschnitte zeigt fast diese ganze Parthie ein die eigenartigsten Formen aufweisendes Netzwerk epithelialer Lumina und Stränge, die zum grossen Theil continuirlich zusammenhängen. Nur spärlich trifft man und dann meist in den peripheren Abschnitten dieses Mucosastückes, seltener inmitten der plexiform angeordneten epithelialen Gebilde annähernd runde Querschnitte von Drüsen, deren einschichtiges Epithel zum Theil hochcylindrische Formen mit basal gestellten einzeiligen cubischen Kernen und spärlichen Becherzellen, zum Theil aber auch niedrig cubische Formen zeigt.

Daneben siebt man auch noch Drüsen, deren eben noch einschichtiges, hochcylindrisches Epithel plötzlich an umschriebener Stelle mehrschichtig wird, wobei zugleich die einzelnen Zellen unter Auftreten s.pärlicher Mitosen polymorphen Charakter annehmen, d. h. kleinere rundliche bis polygonale Formen zeigen. In anderen Drüsen ist es auf diese Weise bereits zur völligen Ausfüllung der Drüsenlumina durch dieses mehrschichtige polymorphe Epithel gekommen.

Während einzelne dieser so umgewandelten Drüsen sich noch vollständig durch ihre Tunica propria gegen das umgebende Stroma abgrenzen lassen, ist dies bei anderen nicht möglich, indem diese 
continuirlich in jenes erwähnte epitheliale Netzwerk übergehen. Wie diese Drüsen, so zeigt auch dieses epitheliale Netzwerk eine starke Polymorphie der einzelnen epithelialen Elemente, indem neben kleinen cubischen Formen plötzlich grosse, bläschenförmige Zellen mit theils runden, theils länglichen und polygonalen Kernen erscheinen; oft auch werden diese epithelialen Stränge durch. mehrere Schichten hoher, an Cervixepithelien erinnernde cylindrischer Zellen gebildet.

Betrachtet man diese Mucosaparthie bei sehwacher Vergrösserung, so lässt sich nicht leugnen, dass auch innerhalb dieser plexiformen Anordnung noch manchmal eine gewisse Andeutang an die Drüsenstructur zu Tage tritt, resp. es den Anschein hat, als ob es sich hier nur um ein Verschmelzen des Epithels der einzeinen Drüsenlängs- und-querschnitte, nicht aber um neugebildete atypische Epithelwucherungen handele. So sieht man häufig diese meist. mehrschichtigen Epithellagen ein unregelmässig gestaltetes Lumen einschliessen und sient in dies Lumen hinein sich sowohl lange, epitheliale mehrsehichtige Bänder, wie auch kurze, knopfartige Erhebungen ohne bindegewebigen Grundstock erheben. An manchen Lumina erscheint oft eine Wandparthie gleichsam überbrückt durch derartige epitheliale Bänder, in anderen wieder sind die knopfartigen, rein epithelialen intraluminaren Erhebungen anscheinend mit ihren freien spitzen verschmolizen, hierdurch das Lumen fächerförmig abtheilend.

An manchen Stellen ist sowohl die plexiforme Anordnung wie auch die Drüsenstructur gesehwunden and es erseheint hier das Epithel nur in Form eines compacten, epithelialen, polymorphen. Gewebes, inmitten des kleinzellig infiltrirten Stroma ausgebreitet. Als solches zeigt es sich auch streckenweise an der freien Fläche des Präparates, doch glaube ich kaum, dass es sich hier um die wirkliche Oberfläche der Mucosa handelt, da stellenweise diesem vielschichtigen, polymorphen Epithel noch eine dünne Bindegewebssehicht aufgelagert ist.

Das Stroma ist nur in der Peripherie dieser Mueosaparthie stärker entwickelt und hier stark kleinzellig infiltrirt. Inmitten des epithelialen Netzwerkes erscheint es nur als äusserst schmale, kleinzellig infiltrirte Septen, die hier auch ausser den scharf gefärbten Rundzellen, mässig reichliche polymorphkernige Leukocyten enthalten. Auch die Lamina des epithelialen Netzwerkes, wie auch innerhalb des Epithels desselben auftretende kleine lacunäre Hohl- 
räume sind zum Theil angefüllt mit rund- und polymorphkernigen Leukocyten, die häufig auch einzelne epitheliale Stränge wie auch besonders die intraluminaren knopfartigen Erhebungen mehr oder weniger dicht infiltriren. Die spärlichen Capillaren sind erweitert und mit roten Blutkörperchen und polymorphkernigen Leukocyten gefüllt.

Da nach dem histologisehen Verhalten eine Schwangerschaft wohl mit Sicherheit auszuschliessen war, auch für Tuberculose keine bestimmten Anhaltspunkte sich ergaben, so stellte ich die Diagnose auf beginnendes glanduläres Carcinom und rieth dem Collegen zur Totalextirpation. Wie ich dann ein Jahr später, gelegentlich eines zufälligen Zusammentreffens mit dem Collegen A. hörte, hat derselbe auch am 26. 11. 1901, 13 Tage nach der Abrasio den Versuch der vaginalen Totalextirpation gemacht, hatte aber nach Unterbindung der einen Uterina wegen starker Blutung die Operation nicht vollenden können. ${ }^{1}$ ) Da die Blutungen nach diesem Eingriff aufhörten, so machte College A., ehe er nochmals einen radicalen Eingriff unternahm, im December desselben Jahres eine zweite Abrasio und sandte das Material zur mikroskopischen Untersuchung an das pathologische Institut nach Göttingen. Von dort erhielt er dann. den überraschenden Bescheid, dass nichts Malignes gefunden sei; denselben negativen Befund ergab dann noch eine dritte am 13. 2. 1902 ausgeführte Abrasio, deren Untersuchung gleichfalls im pathologischen Institut in Göttingen ausgefübrt wurde. Wenn auch ein Zweifel an der Richtigkeit der von mir gestellten Diagnose mir ausgeschlossen schien, so sandte ich doch, um meine anscheinende Fehldiagnose dem Collegen und der Patientin gegenüber zu rechtfertigen, meine Präparate nach Göttingen und erhielt von dem Assistenten des Instituts Herrn Dr. Mundt in Bestätigung meiner Diagnose rom 14. 4. 1902 folgenden Bescheid:

. . . Ihrem Wunsche, die zugesandten Präparate durchzusehen und sie Prof. Orth oder Prof. Aschoff vorzulegen, habe ich gerne entsprochen, um so mehr, als ich trotz eifrigen Bemühens in den Präparaten zweier späterer Ausschabungen nichts Malignes finden konnte. Ich muss in Uebereinstimmung mit Prof. Aschoff (Herr Geheimrath Orth ist noch nicht von der Reise zurückgekehrt) mich

1) Ich kann weder in der eventuell durch die Unterbindung der Uterina eingetretenen trophischen Störung, noch auch in der "starken Blutung" im Sinne Lomer's (20) die Ursache für die Dauerheilung nach der Abrasio erblicken. 
ohne jedes Zögern Threr Diagnose anschliessen. Die von Thnen besonders bezeichnete kleine Parthie trägt entschieden malignen Charakter. Ich habe bei den beiden späteren Untersuchungen, die sich auf sämmtliche durch Ausschabung gewonnene Bröckelchen ( $\mathrm{je}$ 6 grosse Paraffinblöcke) erstreckten, einige wenige Stellen, die sich von dem allgemeinen Bilde etwas zu unterscheiden schienen, Orth zur Begutachtung vorgelegt und meine Diagnose: Endometritis glandularis bestätigt erhalten.

Es handelt sich hier also - vorausgesetzt, dass bei den späteren Abrasionen alle Stellen des Uteruscavum getroffen wurden, - scheinbar um einen Fall, in dem durch eine einmalige Ausschabung ein beginnendes Adenom entfernt wurde. Prof. Aschoff erwähnte heute einen analogen Fall, der denselben Befund gezeigt hätte. Bei dem operativ entfernten Uterus habe sich zu seiner grossen Beschämung keine entsprechende Neubildung, weder makro- noch mikroskopisch nachweisen lassen ....

Natürlich interessirte mich jetzt ansserordentlich von dem späteren Befinden der Patientin zu hören und wandte ich mich dam her, da College A. inzwisehen E. verlassen hatte, direct an die Patientin.

Von dem Ehemann erhielt ich dann im December 1902 den Bescheid, dass seine Frau, trotzdem sie "lungenleidend" sei, 10 Pfund zugenommen habe, dass der Ausfluss fast gänzlich verschwunden und ndas Unwohlsein ganz unregelmässig und ganz schwach" sei, dass weder vor der ersten Ausschabung noch jetzt Schmerze日 bestanden hätten. Am 3. Juni 1903 lautete der Bericht "meine Frau befindet sich bis jetzt vollständig gesand und ist keinerlei Veränderung rorgekommen".

Am 15. Juni 1904 theilte mir der Ehemann mit, dass "seine Frau sich soweit ganz munter and gesund befinde und sich in ihrem Befinden nichts geändert habe und dass die Blatungen bei ihr einen ganz normalen Verlauf bätten". Der letate Bericht ist vom 20. 1. 1905 datirt. Da ich um einen genaueren Krankenbericht aus der Zeit vor der ersten Ansschabung gebeten hatte, schrieb mir der Ehemann "meine Frau hatte s. Zt. beimi Auftreten ihres Unwohlseins einige Male etwas stärkere Blutungen, bis dann auf eimmal grössere Blutungen eintraten, sỏche dauerten 8 Tage und liess sich dann meine Frau einer Aussehabung unterwerfen, wonach die Blutungen sofort aufhörten und bis heute auch nowh nicht wieder aufgetreten sind. Sehmerzen hat moine Fran s. Zt. 
nicht gehabt and war auch nicht Weissfluss vorhanden. Meine Frau befindet sich sonst wohl und tritt ihr Unwohlsein normal auf". Letzte Geburt im Jahre 1895.

Zugleich mit der Anfrage an die Patientin hatte ich mich auch an den Nachfolger des Collegen A. in E. gewandt mit der Bitte, mir einen vollständigen Auszug aus dem Krankenjournal NovemberDezember 1901 zu senden, da naturgemäss mein weiterer Gedanke war, dass eine Verwechslung des Materiales vorgelegen haben könnte, obschon College A. auf mein Befragen, dies für unmöglich erklärt hatte. Auch ich muss nach Einsicht des Journales eine derartige Verwechslung für unmöglich halten und da ich dasselbe mit absoluter Sicherbeit für meine Untersuchung behaupten kann, so kann an der Thatsache, dass das Material der drei verschiedenen Ausschabungen von ein und derselben Patientin stammt, nicht gezweifelt werden.

Eine andere Frage ist die: hat es sich im vorliegenden Fall wirklich um eine maligne Epithelwucherung gehandelt und können wir, bejahenden Falles, die Patientin als dauernd geheilt betrachten?

Um diese zweite Frage gleich vorweg zu beantworten, so glaube ich diese unbedingt bejahen zu können. Denn wenn auch drei Jahre Recidivfreiheit, wofern man diesen Ausdruck für eine Palliativoperation, wie sie die Abrasio dem Carcinom gegenüber unter gewöhnlichen Verhältnissen darstellt, überhaupt gebrauchen darf, noch keineswegs Dauerheilung garantirt, so kann angesichts des negativen Resultates der zwei späteren Ausschabungen und angesichts des späteren guten Allgemeinbefindens bei Fehlen jeglicher localer Symptome, m. E. nicht an der Dauerbeilung im vorliegenden Falle gezweifelt werden. Schwieriger dürfte die Frage zu beantworten sein, $a b$ es sich wirklich um eine maligne Epithelwucherung gehandelt habe.

Differentialdiagnostisch kämen für die Uterusmucosa bezüglich des histologischen Verhaltens vor allem Tuberculose und atypische Epithelwucherung bei regenerativen Processen nach Abrasio und vorausgegangener Schwangerschaft in Betracht.

Eine Abrasio war nicht vorausgegangen und einen Abort können wir histologisch wohl mit Sicherheit ausschliessen. Klinisch liesse sich nach der Anamnese des Collegen A. dieser Verdacht vielleicht aufrecht erhalten, doch spricht $\mathrm{m}$. E. der letzte Bericht der Patientin selbst ebenso bestimmt dagegen. Denn ab- 
geseben davon, dass hier von einem Ausbleiben der Periode nichts berichtet wird, wird ausdrücklich das Fehlen jeglicher, bei unvollständigem $\Delta$ bort doch meist auftretenden Schmerzen sowie Fluors betont; dazu sind die Blutungen anscheinend nur an die Periode gebunden gewesen. In zweiter Linje käme dann eine Tuberculose der Uterusmucosa in Betracht, zumal anscheinend bei der Patientin noch eine tuberculöse Lungenerkrankung vorliegt.

Auch diese lässt sich aber $m$. E. bei dem Fehlen aller für Tubereulose eharakteristischen, histologischen Veränderungen in allen drei Ausschabungen wohl mit ziemlicher Bestimmtheit ausschliessen. Denn wenn bei den Präparaten der ersten Ausschabung auch wohl noch geltend gemacht werden könnte, es hätte eine atypische Form dex Endometritis tuberculosa rorgelegen, wie sie z. B. Schütt (1) und Wallart (2) als rein bacillären Katarrh beschreiben, bei welcher alle histologischen Anzeichen fehlten und nur im pathologischen Secret der Lterusdrüsen sich zahlreiche Tuberkelbacillen fanden oder wie sie Altertum (3) beschreibt, der nur atypisohe Wucherungen fand, so spricht doch m. E. abgesehen davon, dass ich, allerdings nur zwei Schnitte, vergeblich auf Tuberkelbacillen färbte, das vollständige Eehlen aller der erwähnten Veränderungen auch in den späteren Ausschabungen eher gegen als für Tubercullose. Auch das anscheinende Fehlen jeglicher Adnexperänderungen z. Zt. der drei Ausschabungen, sowie das gute Allgemeinbefinden und Fehlen jeglicher Localsymptome von Seiten des Uterus seit jener Zeit dürfte wohl mit Sicherheit gegen Tuberculose sprechen. Es drängt somit alles dazu an unserer. Diagnose einer histologiseh malignen epithelialen Wucherung, die ja anch durch die Autoritat Aschoff's gestützt wird, festzuhalten und es fragt sich nur, wie lässt sich hiermit der weitere günstige klinische Verlauf in Linkllang bringen.

Theoretisch wird ja gewiss von manchem die Möglichkeit einer Radicalheiling des Uterusearcinoms duroh die Ausschabung zagegeben, wenn die carcinomatöse Wucherang noch im frühesten Stadium und aul die Mucosa beschränkt ist. Gessner (4) und v. Franqué(5) haben den anatomischen Beweis für diese theoretische Möglichkeit nachträglich am exstirpirten Uterus zu erbringen versucht - praktisch wird aber, glaube ich, in vielen derartig beginnenden Fällen nicht die Consequenz gezogen und erst das Ergebniss einer zweiten Abrasio abgewartet, sondern meist wohl sofort der Uterus entfernt. 
Auch ich stand früher auf diesem Standpunkt, bin aber seit der Beobachtung dieses Falles sehr viel rorsichtiger in der Deutung aller atypischen Epithelwucherungen in der Cterusmucosa geworden und habe nicht selten erst nach einer zweiten oder sogar dritten Abrasio mich definitiv über die Benignität oder Malignität derartiger Epithelveränderungen auszusprechen gewagt, wie ich gleich hinzufügen möchte, ohne Schädigung der betreffenden Patientinnen. Gerade die Kenntniss der atypischen Epithelwacherangen in der Uterusmucosa bei allen regenerativen Processen und bei der Tuberculose muss uns die Frage vorlegen, ob es nicht unter anderen, uns noch unbekannten Einflüssen zu ähnlichen atypischen Epithelwucherungen, aber gutartiger Natur kommen kann und ob es sich in unserem Falle nicht auch vielleicht um eine derartige gutartige, atypische Epithelwucherung gehandelt habe.

Auffälligerweise ist nun trotz der enormen Häufigkeit, in der die Uterusmucosa mit dem Aufschwung der operativen Gynäkologie Gegenstand der histologischen Untersuchung geworden ist, äusserst wenig über gutartige atypische Epithelwucherung der Uterusmucosa bekannt oder wenigstens beschrieben worden und doch glaube ich nach meinen Erfahrungen an ca. 200 genau untersuchten Ausschabungen seit jenem Falle sagen zu können, dass die dem Pathologen an anderen Organen bei den verschiedensten pathologischen Processen so bekannten atypischen Epithelwucherungen auch in der Uterusmucosa nicht so selten $\sin d^{1}$ ).

Sehon vor Jahren hat Zeller (6) über gutartige Lmwandlung des einsehichtigen cylindrisehen Oberflächenepithels in mehrsehichtiges Plattenepithel berichtet. Doch müsste die Häufigkeit, mit welcher er diese Befunde erhob, - es sollte bei jeder Endometritis hierzu kommen könuen - entschieden die Skepsis herausfordern und wenn man seine Technik der Gewebsentnahme betrachtet, so dürften diese Befunde auch kaum einer ernsten Kritik Stand halten.

Die nächsten Angaben über atypische Epithelwucherungen an der Portio stammen von Ruge und Veit (7) und zwar sahen sie dieselben sehr selten, naber dann in netzförmig angeordneten schmalen Epithelgängen, mitten im stark gereizten, kleinzellig entzündlich

1) Da für manche dieser Fälle die Beobachtungszeit seit der Abrasio zu kurz ist, um auch aus dem klinischen Verhalten die Benignität dieser atypischen Epithelwucherungen sicher garantiren zu können, so behalte ich mir eine geuaue Sebilderung dieser Befunde für eine spätere Arbeit vor. 
infiltrirten Gewebe und zwar entsprechend den entzündeten Stellen meist herdweise." "Es waren nicht diffuse, über grosse Bezirke verbreitete Veränderungen, sondern in Knotenform."

Späterhin vertrat Ruge (8) dann noch auf dem VI. Gynäkologencongress in Wien mit aller Schärfe folgenden Standpunkt:

"Während man bei den Erosionen der Portio bei der Umwandlung des Cylinderepithels in mehrfach geschichtetes Epithel von vollkommenen oder unvollkommenen Heilungsvorgängen sprechen kann, so kann in der Cervix nur von epidermidalisirenden Umwandlungsvorgängen geredet werden. Bei der Endometritis corporis findet sich trotz der grossen epithelialen Wucherungen bei den Entzündungsvorgängen derartige Umwandlungen nicht. Mehrschichtungen an der Oberfläche; wie sie gelegentlich an kleinen Stellen, an kleinen polypösen Wucherungen, auch hier meist circumscript gefunden werden, sind extrem selten. Dass das Cylinderepithel sich vollständig in Plattenepithel umwandeln kann, weiss man (z. B. bei Inversio uteri). Häufig erhobener Befund von mehrfach geschichtetem Plattenepithel (sog. Psoriasis) im Corpus (unter Ausschluss maligner Degeneration) beruht auf Täuschung,"

Demgegenüber machte Wertheim (9) in der an Ruge's Referat sich ansehliessenden Discussion auf eine Erscheinung an der Mucosa in wegen Gonorrhoe erstirpirten Uteris aufmerksam, die schon wiederholt an gonorrhoisch entzündeten Schleimhäuten beobachtet worden ist, "nämlich die Umwandlung des einschichtigen Cylinderepithels in mehrschichtiges Platten- bezw. Uebergangsepithel." "Auch kommt es ror, dass das Epithe] wohl einschichtig geblieben ist, dass es aber mehr kubisch bezw. mehr abgeplattet erscheint."

In neuester Zeit hat dann Mainzer (10) bei nt exstirpirten und früher mit Vaporisation oder unverdünnten Formalinätzungen behandelten Uteris ganz auffälige Netaplasie des Oylinderepithels in gesehichtetes Plattenepithel gesehen. In zweien dieser Fälle hatte die Metaplasie äusserst carcinomverdächtigen Charakter", und Borst (11) wies auf dem letzten Pathologeneongress in seinem Vortrage über atypische Epithelwucherungen und Krebs u. A. darauf hin, dass auch bei den hyperplastischen Vorgängen in der Uterusmucosa unregelmässige Mehrschichtungen des Drüsenepithels, bei welchen natürlich auch die Gestalt der einzelnen Epithelzellen wechselvoll ist, vorkommen, ohne dass damit eine krebsige Umwandlung ohne Weiteres angenommen werden 
dürfe, ja nach Schilderung der bei den verschiedensten Processen und an den verschiedensten Organen beobachteten atypischen Epithelwucherungen nicht carcinomatöser Natur kommt Borst am Schlusse seines Vortrages zu dem Resultat, "dass weder Gestaltsveränderungen der Epithelzellen, noch ungeordnete Aufschichtungen der Epithelzellen, noch die Bildung mehschichtiger Epithelbeläge, noch solide Ausfüllungen der Drüsenlumina mit gewucherten Epithelien bezw. Bildung solider sprossenartiger Fortsätze der D rüsen, noch solide Epithelformationen irgend welcher Art überhaupt - sogar die Membrana propria kann bei allen diesen Veränderungen fehlen bezw. aufgelöst sein - an sich unbedingt für carcinomatös gelten können."

Fast ebenso wichtig, histologisch jedenfalls noch interessanter sind dann schliesslich die von R. Meyer (33), Friedländer (34) und Hoehl (35) an fötalen bezw. kindlichen Uteri erhobenen Befunde von derartigen wahrscheinlich angeborenen Metaplasien des cylindrischen Oberflächenepithels der Uterusmucosa in mehrschichtiges Plattenepithel. Wirft sich doch hier sofort die Frage auf, wie werden sich diese atypischen Epithelbefunde späterhin verhalten, können sie bei späteren Ausschabungen nicht eventuell differenzial-diagnostisch mit in Erwägung kommen müssen. ${ }^{1}$ ) Kehren wir jetzt zu unserem Falle zurück, so muss ich zunächst sagen, dass die dort gefundenen Veränderungen sich weit über das erheben, was ich sonst als atypische Epithelwucherung gefunden habe und dass ich in einem gleichen zweiten Falle zunächst wieder an maligne Wucherung denken und zum mindesten auf eine Wiederholung der Abrasio nach 4 Wochen dringen würde.

Dass derartige Fälle, in denen die Abrasio Carcinom ergab und später am exstirpirten Uterus nichts Carcinomatöses gefunden wurde, nicht so selten sind, sondern nur nicht immer veröffentlicht werden - Gessner (4), Kiefer (12) und Zweifel (13) haben über derartige Fälle berichtet - glaube ich nach mündlicher Mittheilung von befreundeten Gynäkologen und Pathologen sicher annehmen zu können. Nur ist dann die Deutung, glaube ich, meist

1) Wurden doch auch z. B. die an den Papillae vallatae der Zunge auftretenden atypischen Epithelwucherungen noch von Schaffer (36) als "selten zu beobachtende Anfangsstadien einer epithelialen Neubildung gedentet, während Zi ler $_{\ominus}(37)$ nachweisen konnte, „dass es sich hierbei um ganz gewöhnliche, fast regelmässig vorkommende Gebilde handele". 
nicht die, dass es sich entweder nicht um Carcinom gehandelt habe oder dass die Ausschabung alles Carcinomatöse entfernt habe. Denn da bei derartig beginnenden Caroinomfällen natürlich nur eine lückenlose mikroskopische Untersuchung der Uterusmucosa derartige Schlüsse als berechtigt erscheinen lassen kann, eine derartige eingehende Untersuchung aber wohl selten vorgenommen ist, so wird eher geschlossen, dass eben die verdächtige Stello der nachfolgenden Untersuchung am exstirpirten Uterus entgangen sei.

Noch seltener als derartige, einwandsfreie Fälle sind schliesslich Parallelfälle zu dem unsrigen, in denen die Diagnose Carcinom gestellt, die Radicaloperation aber nicht ausgeführt warde und die Patientin trotzdem gesund blieb und leider sind in den wenigen derartigen Fällen, die ich in der Literatur fand, keine mikroskopischen Details mitgetheilt und vor. Allem leider keine Abbildungen veröffentlicht and doch sind gerade diese Fälle so überaus wichtig, sei es zur Revision unserer jetzigen Kenntnisse vom beginnenden Carcinom der Cterusmucosa oder aber um als Beweis für die Möglichkeit einer Dauerheilung desselben durch die Abrasio zu dienen.

So erwähnt Dührssen (14), nachdem er mehrere Male ausgeschabt habe mit dem mikroskopischen Befunde der gutartigen Endometritis glandularis, habe die letzte Abrasio neben diesen Bildern folgendes ergeben An anderen (sc. Drüsen) war nummehr das Epithel gewuchert, zeigte polygonale Formen, in noch weiteren Drüsen war das Lumen völlig mit diesen Zellformen ausgefüllt, so dass hier an Stelle der Drüsenschläuche solide Krebszapfen getreten waren." Während in diesem Falle die Tatalexstirpation gemacht wurde, erwähnt Dührssen weiter, dass er mehrere Fälle gesehen babe, die in threm mikroskopischen Bilde dem beschriebenen völlig glichen und durch ein bezw. mehrere Auskratzungen (1) davernd geheilt wurden," and Opitz (15) hat jüngst äber 2 Fälle aus der Olshausen'schen Klinik berichtet, "bei denen während bestehender Gravidität an Probeexisionen aus der Portio ein histologiseh zweifelloses Carcinom diagnosticirt wurde, während die weitere Beobachtung, da die Operation nicht ausgeführt worde, mit Sicherheit erkennen liess, dass ein Carcinom nicht vorhanden war ${ }^{1}$ ).

1) Ueber einen dritten gleichen Fall berichtot 0 lshausen (Centralblat 1905, S. 114) und erlärt diese Fälle damit, dass in der Schwangerschaft an der Portio adenomatöse Bildungen entstehen können, die spontan wieder zu. rückgeben. 
Während die Dührssen'schen Fälle als Parallelfälle zu dem unsrigen, wie auch der Fall von Krukenberg (16), bei dem nach Exeision eines carcinomatös degenerirten Polypen der vorderen Muttermundslippe anscheinend Dauerheilung eintrat (nach 2 Jahren noch Recidivfreiheit festgestellt) sich auch ähnlich erklären lassen, fehlt mir für die Opitz'schen Fälle, offen gestanden, das Verständniss. Wir müssten dann noch einen Sehritt weiter gehen und annehmen, dass durch die vielleicht sehr ausgiebige Probeexcision zufällig ein ganz beginnendes Carcinom radical exstirpirt sei.. Wird die Erklärung unseres eigenen Falles aber bei Vielen schon auf Zweifel stossen, so wird dieser Zweifel wohl noch grösser und allerdings anch berechtigter sein bei diesem Versuch der Erklä'rung der Opitz'schen Fälle. Viel näherliegend wird für Viele die Annahme sein, dass es sich in allen derartigen Fällen nicht um bösartige epitheliale Wucherungen gehandelt habe.

Wenn auch dieser Zweifel in die Unfehlbarkeit unserer histologischen Diagnose, soweit es sich um die Erkennung der frühesten Stadien einer bösartigen Neubildung zumal in der Uterusmucosa handelt, wohl ron allen Pathologen als berechtigt anerkannt wird - erst jüngst hat $v$. Hansemann auf diese Unsicherheit unserer diagnostischen Kenntnisse hingewiesen, wenn er sagt, dass „was man heutzutage als Carcinom bezeichnet, sicherlich nicht alles gleichwertig ist und dass ganz besonders mit dem Namen Sar$\mathrm{kom}^{1}$ ) noch die allerverschiedensten Geschwulstbildungen bezeichnet werden" -, so muss andererseits doch auch betont werden, dass nach den Erfahrungen der jüngsten Zeit das Dogma von der Unheilbarkeit des Krebses, sofern es nicht gelingt, denselben operativ radikal zu entfernen, nicht mehr haltbar ist und dass es daher auch nicht angängig ist, alle derartigen, mit unseren bisherigen Erfahrungen von der Heilbarkeit bösartiger Neubildungen nicht im Einklang stehenden Fälle von Dauerheilung ohne radikale Eingriffe, einfach mit der Annahme einer mikroskopischen Fehl-Diagnose abzuthun. Haben doch auch Pathologen ron Fach, Orth (18), v. Hansemann (19) jetzt die Möglichkeit einer Radikalheilung

1) Haben doch auch dje unseren bisherigen Anschaungen nach unverständlichen spontanen bezw. auf innere Medication eintretenden Heilungen mancher, bisher als Osteoidsarkome gedeuteten Fälle multipler Knochengeschwülste jugendlicher Individuen wie sie Lallement (25), Rehn (38), Haberer (39), Mikulicz (40) und Wohlgemuth (41) beobachteten, schon dazu geführt, diese "Sarkome" als entzündliche Tumoren aufzufassen. 
nach nur partieller Exstirpation bösartiger Neubildungen anerkannt, nachdem allerdings klinische Erfahrungen schon lange für die Möglichkeit der spontanen Rückbildung bei unvollständigen Operationen zurückgebliebener Krebsmassen gesprochen hatten. Speciell für das Uteruscarcinom hat Lomer (20) kürzlich zahlreiche Fälle aus der Litteratur gesammelt, durch eigene Fälle ergänat und auch versucht diese, nach unseren bisherigen Erfahrungen wunderbaren Heilungen bezw. Rückbildungen za erklären. Auch die chirurgische Litteratur weist derartige Fälle auf, so sagt u. A. Czerny (21) "manche inoperable Carcinome des Uterus konnte ich durch eine combinirte Behandlungsmethode (gründliches Ausschaben mit scharfem Löffel, feste Tamponade, nach gründlicher Austrocknung, mit in 30-50 proc. Chlorzinklösung getauchter steriler Gaze) zur Heilung bringen; ferner weist er auf die auch von Alsberg (23), Krönlein, Lindner, Hahn u. A gemachten Beobachtungen hin, dass nach einfacher Gastro-enterostomie früher fühlbare Magencarcinome verschwänden und die Patienten für viele Jahre hinaus gesund geblieben sind und schliesst aus diesen Beobachtungen, „dass zweifellos lrrebsartige Erkrankungen manchmal einer spontanen Rückbildung fähig sind und dass selbst ganz verzweifelte inoperable Fälle von bösartigen Neubildungen dureh eine combinirte, zielbewusste Behandlung einer Heilung zugängig sind ${ }^{\text {t. }}$ Schuchardt (22) and Alsberg (23) sahen eine peritoneale Oarcinose nach Laparotomio vollständig heilen, Rotter (24) ein malignes Adenom des Rectum, welches schon ins Vaginalgewölbe durchgewuchert war, sich spontan zurïckbilden, trotzdem nach Exstirpation des Rectum und mehrmaliger Auslöffelung von Recidivmassen der Fall als inoperabel entlassen war. Ferner beschreibt Lallement (25) u. A. spontane Heilung eines Recidivs nach Skirrhus mammae. Martin (26) erwähnt einen Fall von mikroskopiseh sicher gestelltem Skirrhus der Portio, in welchem das ganze Scheidengewölbe und Collum in einen Krater verwandelt war and der, trotzdem die Operation verweigert wurde, nach 22 Jahren noch lebte und keiner ärztlichen Behandlung bedurfte, und Senger (27) hat kürzlich über einen. Fall von mikroskopisch sicher gestelltem Wangenschleimhautearcinom, entstanden durch Reibung an einem spitzen Zahnstumpf, berichtet, in welchem er die eine Hälfte der Geschwulst exstirpirte und zugleich auch den Zahnstrmpf entfernte, worauf das zurüekgelassene Stück spontan zurückging und rölige Heilung eintrat. Einer ähnlichen Fall berichtet auch Mohr (28). 
Als weiteren Beweis für die Möglichkeit der spontanen Rückbildung bezw. des Unterganges verschleppter Partikel bösartiger Neubildungen weist Petersen (29) ferner mit Recht darauf hin, dass trotz des nach Goldmann's (30) Untersuchungen wohl häufigen Einbruchs von Carcinommassen in die Blutbahn, doch selten Blutmetastasen sich finden, diese vielmehr, wie auch M. B. Schmidt (31) direct nachweisen konnte, hier in ausgedehntem Maasse zu Grunde gehen müssen.

Natürlich werden durch diese Beobachtungen spontaner Rückbildung oder selbst vollständiger Ausheilung bösartiger Neubildungen nach nur partieller Exstirpation derselben, Fälle wie der unsrige nicht erklärt, sondern hier kann es sich nur um die Frage handeln, kann durch die Abrasio eine beginnende, auf die Mucosa beschränkte bösartige epitheliale Wucherung radikal entfernt werden, und da wir diese Frage aus anatomischen Gründen unbedingt bejahen können, so steht auch der Annahme einer Dauerheilung durch diesen Eingriff unter diesen besonderen Umständen nichts im Wege, denn wie Virchow (32) schon vor Jahren betonte „ist der Krebs in seinem Beginn und oft noch sehr lange ein örtliches Leiden, so muss es auch möglich sein, ihn in dieser Zeit örtlich zu heilen."

\section{Literatur.}

1. Schütt, Reine bacilläre Erkrankung epithelbedeckter Flächen bei primärer Tuberculose des Urogenitalapparates. I.-D. Kiel. 1889.

2. Wallart, Ueber die Combination von Carcinom und Tuberculose des Uterus. Zeitschr. f. Geb. u. Gyn. Bd. 50. S. 243.

3. Alterthum, Tuberculose der Tuben und des Beckenbauchfells. Hegar's Beiträge zur Geb. u. Gyn. Bd. I. S. 42.

- Derselbe, Zar Pathologie und Diagnose der Cervixtuberculose. Centralbl. für Gyn. 1902. S. 197.

4. Gessner, Ueber den Werth und die Technik des Probocurettements: Zeitschr. f. Geb. u. Gyn. Bd. 34. S. 387.

5. v. Franqué, Ueber Endometritis, Dysmenorrhoe und Abrasio mucosae. Zeitschr. f. Geb. u. Gyn. Bd. 38.

6. Zeller, Plattenepithel im Uterus (Psoriasis uterina). Dieses Archiv. Bd. XI. S. 56.

7. Ruge und Veit, Der Krebs der Gebärmutter. Zeitschr. f. Geb. u. Gyn. Bd. 7. S. 138 .

8. Ruge, Endometritis cervicalis mit besonderer Berücksichtigung der Laceration, des Ectropiums, der Endometritis und der Erosion. Verh. der; Deutschen Gesellschaft für Gynälrologie. Wien. 1895. S. 180.

9. Wertheim, ebenda, S. 208. 
10. Mainzer, Centralblatt für Gyn. 1903. S. 609 ,

11. Borst, Ueber atypische Epithelwucherungen und Krebs. Verh. der D. path. Gesellschaft I. Berlin. 1904. S. 110.

12. Kiefer, Zeitschr. f. Geb. u. Gyn. Bd. 34. S. 374.

13. Zweifel, Deutsche med. W. 1903. V.-B. S. 30.

14. Dührssen, Zeitsohr. f. Geb.u. Gyn. Bd. 31 .

15. Opitz, Zwei nngewöhnljche Uteruscarcinome nebst Bemerkungen zun Theorie der bösartigen Geschwülste. Zeitschr. f. Geb. u. Gyn. Bd. 49. S. 169.

16. Krukenberg; Zwei neue Fälle von Adenoma malignum der Cervixdrüsen. Monätsschr. f. Geb. u. Gyn. Bd. V. S. 138.

17. v. Hansemann, D. med, W. 1904. S. 119.

18. Orth, Ueber Heilungsvorgänge an Epitheliomen nebst allgemeinen Bemerkungen über Epitheliome。 D. med. W. 1904. V, -B. S. 126\%.

19. v. Hansemann, ebenda.

20. Lomer, Zur Frage der Heilbarkeit des Carcinoms." Zeitschr. f. Geb. und Gyn. Ba. 50. S. 305 .

21. Czerny, Warum dürfen wir die parasitäre Theorie für die bösartigen Gesobwülste nicht aufgeben?. Beiträge zur klin. Chir. Bd. 25. S. 243.

22. Schuchardt, Sollen wir das, Carcinoma uteri paravaginal oder abdominal operiren? Verh, der D, Gesellschaft für Gyn. Giessen. 1901. S. 126.

23. Alsberg, Casuistische Beiträge zur Chirurgie des Magencarcinoms. Münchn. med. W. 1896. S. 1225.

24. Rotter, Polyposis recti. Adenoma malignum - Spontanheiltug. Archiø für klin. Chir. Bd. 58. S. 357 .

25. Primislar-Lallement, De l'amélioration et de la guếrison spontanées du cancer. Thèse de Bordeaux. 1903.

26. Martin, Zar Behandlung des sog. inoperablen Uteruscarcinoms. Ther. d. Gegenwart. 1904. S. 241 .

27. Senger, Ueber die spontane Hellbarkeit des Carcinoms. Chirurgencongress.' 1904.

28. Mohr, Ueber spontane Heilungsvorgänge beim Carcinom. Ther. Monatshefte." 1903. S. 552 u. 617.

29. Petersen, Beiträge zur Lehre vom Carcinom. II. Ueber Heilungsvorgänge im Carcinom, zugleieh cin Beitrag zur Renntniss der Carcinomriesenzellen. Beiträge zur hlin. Chir. Bd. 34. S. 682.

30. G.old mann, Anatomische Untersuchtngen tiber die Verbreitungswege bös. artiger Geschwülste. Beiträge zur klin. Chir. Bd, 18. S. 595.

31. M. B. Schmidt, Die Yerbreitungswege der Carcinome und die Beziehung generalisirender Sarkome za den leakämisohen Neubildungen. Festschrift für tr. Fecklinghausen. Ref. Dentsohe med. W. 1904. S.1177.

32. Virch ow, Zur Diagnose und Prognose des Carcinoms. Virehows Arehiy. Bd. WI.. S. 24:

33. R. Meyer, Ueber die fötale Uterusschleimbaut. Zeitschrift für Geb. und Gyn. Bd. 38 . S. 247.

34. Friedläuder, Abnorme Epithelbildungen im kindlichen Uteras. Zeitschr. f. Geb. u. Gyn. Bd. 38. 
35. Hoehl, Abnorme Epithelbildungen im kindlichen Uterus. Monatsschr. f. Geb. u. Gyn. Bd. 13. S. 61.

36. Schaffer, Wiener Sitzungsberichte. Abth. III. Bd. 106. Cit. bei Zieler.

37. Zieler, Ueber atypische epitheliale Bildung in den umwallten Zungenpapillen des Menschen. D. med. W. 1901. S. 171.

38. Rehn, Chirurgencongress 1904.

39. Haborer, ref. Ther. d. Gegenwart. 1904. S. 508.

40. Mikulicz, ebenda.

41. Wohlgemuth, ebenda.

\section{Erklärung der Abbildungen anf Tafel XVII.}

Die Photogramme

Ia und IIa stellen Schnitte durch 2 verschiedene Ebenen der carcinomatösen Mucosapartie dar. Ocul. III. Obj. III. Vergr. ca 108 fach.

Ib entspricht Ia bei ca. 72 facher Vergrösserung.

IIb entspricht IIa bei ca. 126 facher Vergrösserung mit gleichzeitiger Verschiebung des Schnittes nach. links und unten, um einzelne der annähernd noch normalen Drüsenquerschnitte rechts seitlich im Photogramme zur Darstellung zu bringen. Ocul. IV. Obj. III. Vergr. ca. 126 fach.

III entspricht der Stelle X im Photogramme Ilb. Ocul. IV. Obj. VIII. Vergr. ca. $700 \mathrm{fach}$. 
ia

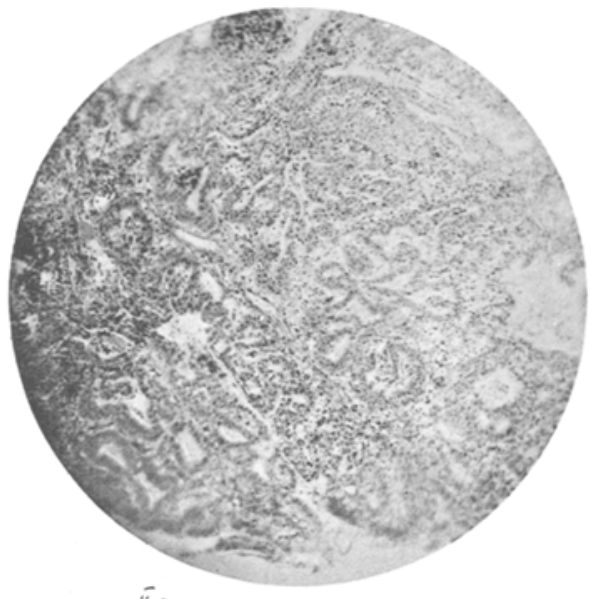

"a

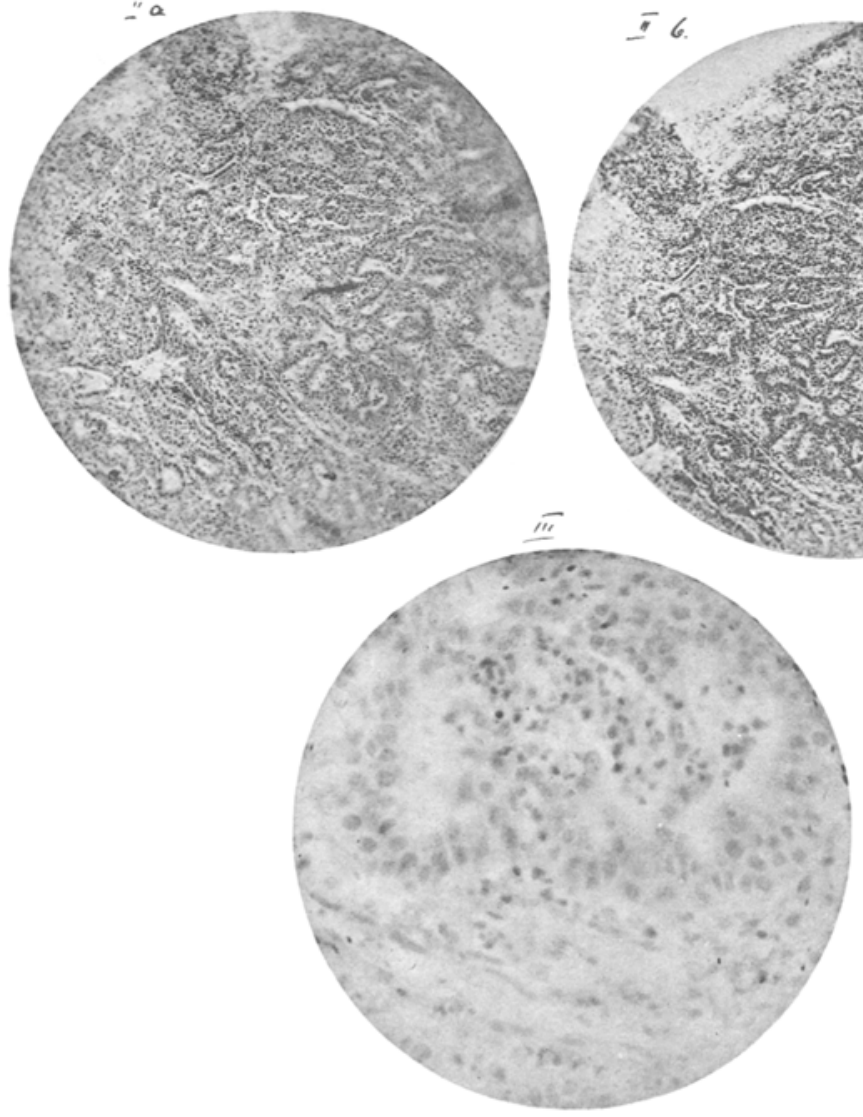

$-$
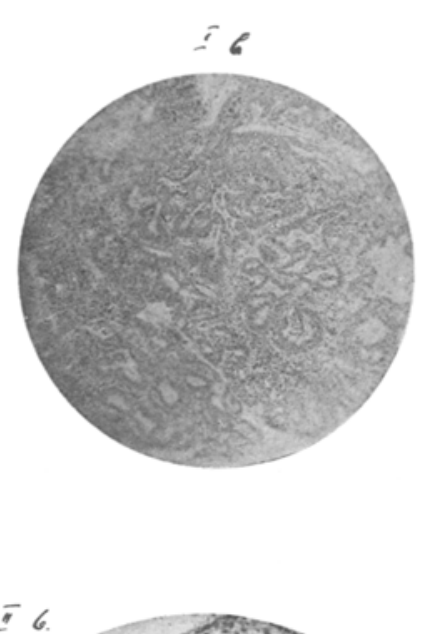

gatco
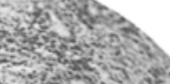

$43 y^{2}+250$

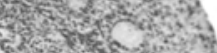

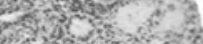
andery



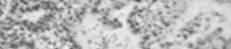
$2 x^{2}+x^{2}+2$

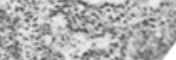
( 2. *..
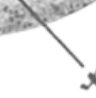